\title{
VALOR E VARIAÇÕES DA FREQÜÊNCIA FUNDAMENTAL NO CHORO DE DOR DE RECÉM-NASCIDOS
}

\author{
Value and variations of fundamental \\ frequency in newborn pain cry
}

\author{
Anete Branco (1), Saskia Maria W. Fekete (2), \\ Lígia Maria S. de Souza Rugolo ${ }^{(3)}$, Maria Inês Rehder ${ }^{(4)}$
}

\begin{abstract}
RESUMO
Objetivo: estudar o valor da freqüência fundamental e suas variações presentes no choro de dor de recém-nascidos. Métodos: foram gravadas as emissões de 111 recém-nascidos de termo e saudáveis, com idade de 24 a 72 horas durante procedimento da punção venosa periférica. A análise acústica foi realizada por meio dos softwares VOXMETRIA 1.1 com extração do valor da freqüência fundamental e GRAM 5.7 para verificar a ocorrência de variações da freqüência fundamental como quebras, bitonalidade e freqüência hiperaguda. A escala de dor NIPS foi realizada no momento da punção. A análise estatística é descritiva com extração dos valores de média, desvio-padrão e freqüência de ocorrência dos eventos. Resultados: os recém-nascidos apresentaram 100\% de suas emissões com variações de freqüência, ou seja, quebras e bitonalidade. A freqüência hiperaguda foi encontrada em $34,2 \%$ dos recém-nascidos. Conclusão: por meio do choro, o recém-nascido comunica sua dor. A emissão de dor do recém-nascido é tensa e estridente, com freqüência fundamental aguda e variações encontradas no traçado espectrográfico, como quebras, bitonalidade e freqüência hiperaguda. Tais características são importantes para chamar a atenção do adulto no pronto atendimento ao recém-nascido e auxiliar na avaliação de dor durante um procedimento.
\end{abstract}

DESCRITORES: Acústica da Fala; Choro; Dor; Recém-Nascido

\section{INTRODUÇÃO}

O choro do recém-nascido é definido como um fenômeno complexo que ocorre durante a fase expiratória da respiração. Para haver o choro, é necessário correto funcionamento dos músculos supralaríngeos, laríngeos e respiratórios ${ }^{1}$, além dos faciais, faríngeos, da boca e do tronco ${ }^{2}$. Essa ação sinérgica de músculos, nervos e estruturas é decorrente do controle do sistema nervoso que garante estabilidade e coordenação laríngea.

(1) Fonoaudióloga; Professora da Clínica Parole de Fonoaudiologia; Mestre em Pediatria pela Faculdade de Medicina de Botucatu da Universidade Estadual Paulista.

(2) Médica, Professora Assistente Doutora do Departamento de Pediatria da Faculdade de Medicina de Botucatu da Universidade Estadual Paulista; Doutora em Pediatria pela Faculdade de Medicina de Botucatu da Universidade Estadual Paulista.

(3) Médica, Professora Assistente Doutora do Departamento de Pediatria da Faculdade de Medicina de Botucatu da Universidade Estadual Paulista; Doutora em Pediatria pela Faculdade de Medicina de Botucatu da Universidade Estadual Paulista.

(4) Fonoaudióloga, Professora do Curso de Especialização em Fonoaudiologia Clínica; Doutora em Distúrbios da Comunicação Humana pela Universidade Federal de São Paulo.
A freqüência fundamental é determinada pela tensão das pregas vocais pelos músculos da laringe que são controlados pelo nervo vago (X par craniano), sendo a coordenação laríngea caracterizada, então, pela variabilidade desta freqüência fundamental ${ }^{3}$.

O som laríngeo produzido nas pregas vocais será modificado pelas estruturas do trato vocal, que no recém-nascido, se apresenta com características particulares. Ao nascimento, a posição da laringe é alta, iniciando sua descida no pescoço ao mesmo tempo em que o trato vocal modifica suas configurações.

O recém-nascido possui pregas vocais medindo 2,5 a 3 milímetros $(\mathrm{mm})$ de comprimento, mucosa espessa, ligamento vocal imaturo e fibras musculares finas e pouco desenvolvidas ${ }^{4}$, a laringe tem forma de funil com cartilagens delicadas e flexíveis ${ }^{5}$.

Autores ${ }^{6,7}$ sugerem que o controle voluntário do choro é adquirido após um mês de idade e durante este tempo, o choro da criança reflete vários estados psicofisiológicos como fome, sofrimento e dor. Conforme o sistema nervoso amadurece e o ambiente da criança se torna mais complexo, o choro deixa de ser um ato reflexo e torna-se um resultado da real atividade volitiva. 
Isso acontece também em relação à dor do recém-nascido. À medida que as respostas aos estímulos dolorosos vão se desenvolvendo, a dor se torna mais organizada e consistente ${ }^{8}$ juntamente com as respostas vocais da criança.

A expressão de dor no recém-nascido, após um estímulo doloroso, é caracterizada pela emissão do choro em conjunto com modificações faciais e corporais $^{9-13}$, além de reações fisiológicas de intensidade e características variáveis ${ }^{13-15}$.

As primeiras descrições acústicas dos diferentes tipos de choro do recém-nascido foram realizadas por pesquisadores nórdicos na década de $60^{16,17}$. Eles descreveram que o choro de dor é o de maior duração com melodia ascendente-descendente, com freqüência de $530 \mathrm{Hertz}(\mathrm{Hz})$, estridente e com queda de sustentação.

Por meio da extração dos valores da freqüência fundamental e suas variações em diferentes situações, torna-se possível obter informações sobre o funcionamento das estruturas vocais e como o recém-nascido reage frente a estímulos dolorosos. Auxilia, portanto, a avaliação da dor e na conseqüente opção por diferentes técnicas e procedimentos, doses de analgésicos, uso de métodos alternativos ou aconchegos, que podem aliviar a dor do recémnascido.

O objetivo deste trabalho é estudar o valor da freqüência fundamental e suas variações presentes no choro de dor de recém-nascidos.

\section{MÉTODOS}

Participaram deste estudo, 111 recém-nascidos a termo, saudáveis e de ambos os gêneros submetidos a punção venosa periférica entre 24 a 72 horas de vida.

Foram excluídos recém-nascidos asfíxicos, com infecções e malformações congênitas e alterações neurológicas diagnosticadas nas primeiras 24 horas de vida.

A punção venosa periférica foi realizada por auxiliares de enfermagem, como procedimento de rotina a fim de coletar amostras de sangue para sorologia. No momento imediatamente anterior à punção, nenhum recém-nascido estava chorando e também não receberam nenhum tipo de abordagem para o controle da dor. Durante o procedimento, médicos residentes em Neonatologia aplicaram a escala de dor NIPS - Neonatal Infant Pain Scale ${ }^{18}$ avaliando expressão facial, choro, respiração, movimentos de braços e pernas e estado de alerta do recém-nascido.

A punção venosa periférica foi realizada com 49 horas de vida com tempo de jejum da última mamada de 38,4 minutos, durando em média 2,8 segundos.

Os dados da amostra foram os choros dos recém-nascidos durante o procedimento da punção ve- nosa periférica, os quais foram coletados por meio de minidisc portátil (SONY - MZ NH700) com microfone externo (AIWA - DM H15) a dez centímetros (cm) de distância da boca do recém-nascido.

Para a análise dos dados, foram utilizados os programas acústicos VOXMETRIA 1.1 (CTS Informática) para extração da freqüência fundamental e GRAM 5.7 (Voice Tools Inc) para descrição de variações, quebras e instabilidade da freqüência fundamental e freqüência hiperaguda por meio de leitura do espectrograma.

A presente pesquisa foi avaliada pelo Comitê de Ética e Pesquisa da Faculdade de Medicina de Botucatu, aprovada sob o número 169/2004 considerada como sem risco e com necessidade do termo de consentimento livre e esclarecido.

$\mathrm{Na}$ análise estatística para descrição da amostra, foram calculados valores da média e seu respectivo desvio-padrão para cada uma das variáveis pertinentes, considerando a freqüência dos eventos ${ }^{19}$.

\section{RESULTADOS}

A amostra é composta por recém-nascidos de ambos os gêneros em partes iguais, com peso adequado para a idade $(73,9 \%)$. Os resultados antropométricos evidenciam valores esperados para a idade estudada com média de 3009,7 gramas de peso, $47,8 \mathrm{~cm}$ de estatura e $33,9 \mathrm{~cm}$ de perímetro cefálico. A idade gestacional de 270,13 dias confirma a amostra sendo recém-nascidos a termo.

Os recém-nascidos estudados apresentaram exames neurológicos e físicos normais, confirmando a homogeneidade de recém-nascidos saudáveis. As características da amostra podem ser conhecidas na Tabela 1.

Todos os recém-nascidos deste estudo choraram durante a punção venosa periférica.

Os valores da Escala de Dor NIPS estão relacionados na Tabela 2.

$\mathrm{Na}$ Tabela 3, são encontrados os valores e a ocorrência da freqüência fundamental no choro dos recémnascidos submetidos a punção venosa periférica. $O$ valor médio da freqüência fundamental é $546,2 \mathrm{~Hz}$ e as variações da freqüência encontradas são quebras e bitonalidade em $100 \%$ dos recém-nascidos da amostra (Figura 1). A freqüência hiperaguda foi observada em $34,2 \%$ dos recém-nascidos (Figura 2). 
Tabela 1 - Características dos recém-nascidos relacionadas ao sexo, adequação do peso para idade, medidas antropométricas e dados da punção venosa periférica com seus valores em porcentagem, médias e seus respectivos desvio-padrão

\begin{tabular}{lc}
\hline & $\begin{array}{c}\text { Valores: } \\
\text { porcentagens e } \\
\text { médias }\end{array}$ \\
\hline Dados do recém-nascido & \\
Gênero & \\
$\quad$ Masculino (\%) & 51,4 \\
Feminino (\%) & 54,6 \\
Adequação do peso para & \\
idade & \\
PIG (\%) & 21,6 \\
AIG (\%) & 73,9 \\
GIG (\%) & 2,7 \\
Peso (g) & $3009,7 \pm 476,32$ \\
Estatura (cm) & $47,87 \pm 2,26$ \\
Perímetro cefálico (cm) & $33,90 \pm 1,89$ \\
Idade gestacional (dias) & $270,13 \pm 8,82$ \\
\hline Dados da punção venosa periférica \\
Idade pós-natal (horas) \\
Tempo de jejum (min) & $49,36 \pm 19,07$ \\
Tempo de duração & $38,48 \pm 36,60$ \\
(min) & $2,76 \pm 1,67$ \\
\hline
\end{tabular}

PIG: pequeno para idade gestacional; AIG: adequado para idade gestacional;

GIG: grande para idade gestacional; g: gramas; $\mathrm{cm}$ : centímetros; min: minutos

Tabela 2 - Freqüência do escore da Escala NIPS de recém-nascidos submetidos à punção venosa periférica

\begin{tabular}{lcc}
\hline Escore da escala NIPS & N & $\%$ \\
\hline 0 & 3 & 2,7 \\
1 & 2 & 1,8 \\
2 & 4 & 3,6 \\
3 & 8 & 7,2 \\
4 & 10 & 9,0 \\
5 & 13 & 11,7 \\
6 & 31 & 27,9 \\
7 & 40 & 36,0 \\
\hline
\end{tabular}

Tabela 3 - Valor médio da freqüência fundamental e seu respectivo desvio-padrão e ocorrência encontrada de variações da freqüência

\begin{tabular}{lccc}
\hline Parâmetros acústicos & & \\
& Valor médio da freqüência fundamental (Hz) & & \\
e desvio-padrão & & & \\
& $\mathbf{N}$ & $\%$ \\
& 111 & 100 \\
Variações da freqüência & 111 & 100 \\
quebras & 38 & 34,2 \\
bitonalidade & & \\
hiperaguda
\end{tabular}

$\mathrm{Hz}$ : hertz 


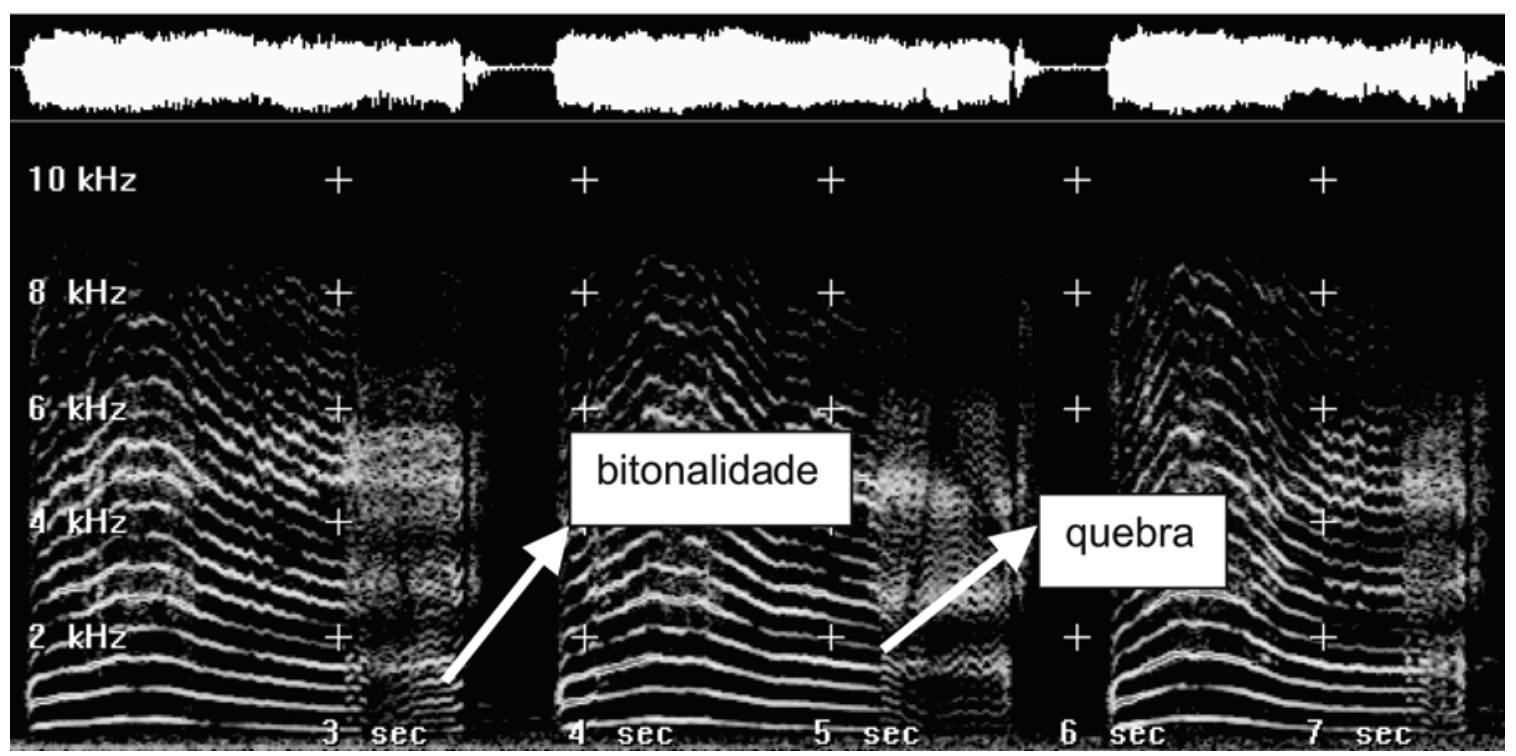

Figura 1 - Espectrograma do choro de recém-nascido durante punção venosa periférica (observar quebras e bitonalidade da freqüência)

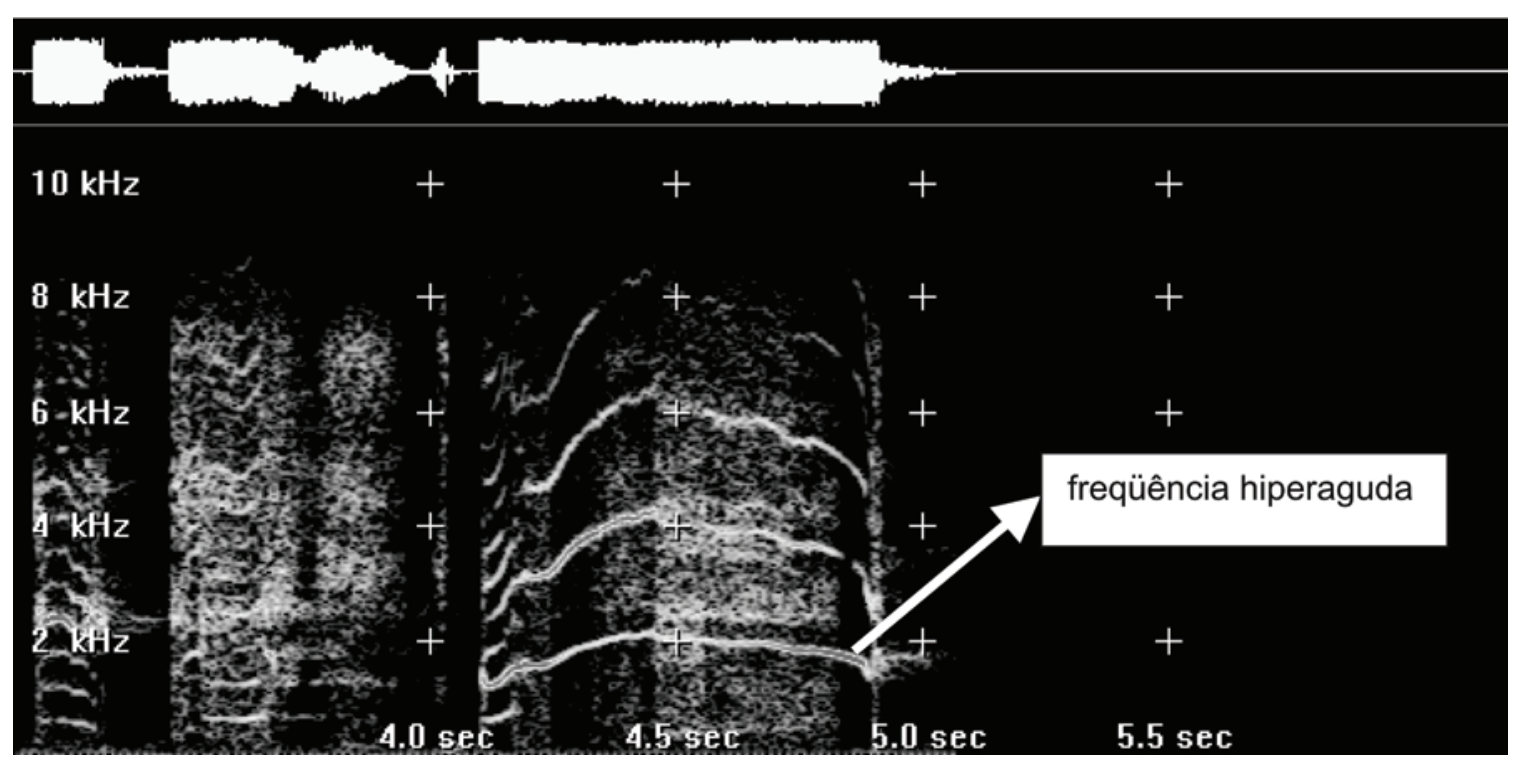

Figura 2 - Espectrograma do choro de recém-nascido durante punção venosa periférica com presença de freqüência hiperaguda

\section{DISCUSSÃO}

Desde o nascimento, o choro é um sinal de comunicação entre o recém-nascido e o ambiente, denotando necessidades emocionais e fisiológicas como fome e dor. Em unidades de cuidados neonatais, as experiências dolorosas fazem parte dos cuidados da criança doente e muitos procedimentos são realizados sem preocupação dos profissionais em administrar anestesia ou analgesia adequadas, pois o recémnascido não verbaliza a dor ${ }^{20}$.

Como visto na Tabela 1, os recém-nascidos deste estudo são a termo e saudáveis e choraram durante o procedimento da punção venosa periférica igualmente encontrado em outro estudo ${ }^{8}$ e ausentes em $50 \%$ da amostra pesquisadas por outros autores ${ }^{21,22}$.

As emissões encontradas aqui são consideradas normais e íntegras em relação ao estado neurofisiológico do recém-nascido ${ }^{23,24}$.

O tempo de jejum entre a última mamada e a punção venosa periférica foi de aproximadamente 40 minutos, sugerindo que os recém-nascidos não estavam com fome. Na literatura, há vários estudos que diferenciam o choro de dor com o de fome, sendo o de fome composto de rápidas variações de freqüência, sons plosivos e mais curtos ${ }^{17}$; já o de dor, é 
mais forte possuindo o segundo formante com maior amplitude ${ }^{25}$.

A dor do recém-nascido deve ser analisada por meio de medidas multidimensionais, combinando variáveis objetivas e subjetivas, somando ao contexto ambiental em que se encontra o recém-nascido, permitindo avaliação válida da presença da dor ${ }^{26}$. A Escala NIPS é útil na avaliação da dor, diferenciando estímulos dolorosos dos não-dolorosos ${ }^{22}$.

No presente estudo, $85 \%$ dos recém-nascidos sentiram dor durante a punção venosa periférica, apresentando média do escore da Escala NIPS acima de 4 (Tabela 2). Tal resultado pode estar relacionado ao avaliador, que neste caso, foram médicos residentes em Neonatologia em treinamento.

Na avaliação de dor de recém-nascidos, a interpretação das respostas comportamentais pode ser diferente entre os avaliadores ${ }^{27}$, explicando uma visão individual das respostas dos recém-nascidos frente à dor.

Crianças recém-nascidas apresentam valores de freqüência fundamental de aproximadamente $300 \mathrm{a}$ $600 \mathrm{~Hz}$. Fortes desvios nestas características como aumento significativo da variação da freqüência, mudança súbita da freqüência fundamental ou sons vocálicos, podem estar relacionados a severos estados patológicos do sistema do controle cerebral ${ }^{3,28}$.

A freqüência fundamental média encontrada no presente estudo foi $546,2 \mathrm{~Hz}$ (Tabela 3), ou seja, em recém-nascidos durante um procedimento doloroso, a freqüência é aguda corroborando com vários estudos que avaliaram este parâmetro 8,12,17,29,30.

O valor da freqüência nos recém-nascidos submetidos a procedimento doloroso, como a punção venosa periférica é diferente dos valores da freqüência no momento do nascimento. Recém-nascidos de partos cesáreas apresentam freqüência fundamental média de $382,1 \mathrm{~Hz}$ e os de parto normal, $371,8 \mathrm{~Hz}^{2}$, com isso, o valor médio da freqüência fundamental encontrado no presente estudo é característico de uma situação dolorosa.

O aumento do valor da freqüência fundamental pode estar relacionado com a diminuição do tônus vagal conseqüente da resposta do recém-nascido frente ao estresse ${ }^{31}$ durante um procedimento doloroso.

Outro fator importante relacionado à freqüência fundamental são suas variações encontradas no espectrograma. Nas Figuras 1 e 2, pode-se observar variações como bitonalidade, quebras e freqüência hiperaguda.

Todos os recém-nascidos deste estudo apresentaram quebras e bitonalidade de freqüência (Tabela 3). Tal resultado corrobora com pesquisa anterior ${ }^{12}$ que enfatiza o ciclo do choro composto por quebras e deslocamento de freqüência em vários pontos da emissão.

Embora a bitonalidade seja descrita como anormal quando associada a freqüências altas ${ }^{1}$, ela está presente nas emissões dos recém-nascidos sob pro- cedimento doloroso, como os do presente estudo. Tal achado também é descrito na literatura ${ }^{32}$, denotando que a bitonalidade pode ser uma característica do choro de dor, decorrente da tensão provocada pela inibição do controle neural ${ }^{33}$.

A freqüência hiperaguda, ou seja, o aumento da freqüência fundamental, foi encontrada em 38 recémnascidos.

Autores ${ }^{34}$ referem que a freqüência hiperaguda está associada à tensão da musculatura decorrente de estresse, e há relatos de estar presente em procedimentos dolorosos ${ }^{35}$.

A freqüência hiperaguda também pode ser encontrada em doenças congênitas e do sistema nervoso ${ }^{1,32}$.

No presente estudo, os recém-nascidos são saudáveis e sentiram dor de maneira individual e a presença de freqüência hiperaguda pode ser uma característica da emissão dolorosa por aumento de tensão.

A busca por métodos mais objetivos e eficientes de avaliação de dor no período neonatal tem levado pesquisadores e cuidadores a valorizarem diversas respostas do recém-nascido durante procedimentos considerados dolorosos. Dentre elas, o choro chama atenção por sua variedade de sons e características acústicas. Atualmente, a análise do choro já faz parte de inúmeras escalas de avaliação de dor, sendo importante a continuação e aprofundamento de seu estudo em diferentes situações.

\section{CONCLUSÃO}

O choro é uma das formas que o recém-nascido utiliza para comunicar sua dor. A emissão de dor do recém-nascido é tensa e estridente, com freqüência fundamental aguda e variações encontradas no traçado espectrográfico, como quebras, bitonalidade e freqüência hiperaguda. Tais características tornam o choro de dor peculiar auxiliando na avaliação de dor durante um procedimento e na conseqüente opção por diferentes técnicas e procedimentos a fim de aliviar a dor dos recém-nascidos. 


\begin{abstract}
Purpose: to study the value of fundamental frequency and its variations found in newborn pain cry. Methods: emissions of 111 healthy and term newborns were recorded, whose lifetime varied from 24 to 72 hours, during venepuncture procedures. The acoustic analysis was realized with VOXMETRIA 1.1 software, with an extract from the fundamental frequency value and GRAM 5.7, in order to verify the occurrence of fundamental frequency variations such as breaks, double harmonic breaks and higher fundamental frequency. The NIPS scale was carried out during venepuncture. The statistical analysis is descriptive with extract from the mean values, standard deviation and frequency of event occurrence. Results: the newborns showed $100 \%$ of their emissions with frequency variations, in other words, breaks and double harmonic breaks. The higher fundamental frequency was found in $34.2 \%$ of the newborns. Conclusion: the newborn communicates pain through cry. The newborn pain emission is tense and strident with higher fundamental frequency and variations founds at the spectrographic tracing such as breaks, double harmonic breaks and higher fundamental frequency. Such characteristics are important to attract adult attention concerning as for a fast support to the newborns and to help in the evaluation of pain during a procedure.
\end{abstract}

KEYWORDS: Speech Acoustics; Crying; Pain; Infant, Newborn

\section{REFERÊNCIAS}

1 - Michelsson K, Michelsson O. Phonation in the newborn, infant cry. Int J Pediatr Otorhinolaryngol. 1999; 49(suppl 1):S297-301.

2 - Branco A, Behlau M, Rehder MI. The neonate cry after cesarean section and vaginal delivery during the first minutes of life. Int J Pediatr Otorhinolaryngol. 2005; 69(5):681-9.

3 - Lind K, Wermke K. Development of the vocal fundamental frequency of spontaneous cries during the first 3 months. Int J Pediatr Otorhinolaryngol. 2002; 64(2):97-104.

4 - Hirano M, Kurita S, Nakashima T. Growth, development and aging of human vocal folds. In: Bless DM, Abbs JH. Vocal fold physiology. San Diego: College-Hill; 1983. p. 23-43.

5 - Kahane J. Lifespan changes in the larynx: an anatomical perspective. In: Brown WS, Vinson BP, Crary MA, editors. Organic voice disorders: assessment and treatment. San Diego: Singular; 1996. p. 89-110.

6 - Robb MP, Goberman AM, Cacace AT. An acoustic template of newborn infant crying. Folia Phoniatr Logop. 1997; 49(1):35-41.

7 - Wermke K, Mende W, Manfredi C, Bruscaglioni P. Developmental aspects of infant's cry melody and formants. Med Eng Phys. 2002; 24(7-8):501-14.

8 - Johnston CC, Stevens B, Craig KD, Grunau RV. Developmental changes in pain expression in premature, full-term, two-and four-month-old infants. Pain. 1993; 52(2):201-8.

9-McGrath PA. Pain in children: nature assessment and treatment. New York: The Gilford Press; 1990.

10 - Craig KD, Whitfield MF, Grunau RV, Linton J,
Hadjistavropoulos HD. Pain in the preterm neonate: behavioural and physiological indices. Pain. 1993; 52(3):287-99.

11 - Stevens B. Pain management in newborns: how far have we progressed in research and practice? Birth. 1996; 23(4):229-35.

12 - Runefors P, Arnbjörnsson E, Elander G, Michelsson K. Newborn infants' cry after heel-prick: analysis with sound spectrogram. Acta Paediatr. 2000; 89(1):68-72.

13 - Stevens BJ, Franck LS. Assessment and management of pain in neonates. Paediatr Drugs. 2001;3(7):539-58.

14 - Guinsburg R. Dor no recém-nascido. In: Rugolo LMSS, editora. Manual de neonatologia. 2. ed. Rio de Janeiro: Revinter; 2000. p. 63-9.

15 - Branco A, Fekete SMW, Rugolo LMSS. O choro como forma de comunicação de dor do recém-nascido: uma revisão. Rev Paul Pediatr. 2006; 24(3):270-4. 16 - Sedlácková E. Development of the acoustic pattern of the voice and speech in the newborn and infant. Prague: Academia; 1967.

17 - Wasz-Höckert O, Lind J, Vuorenkoski V, Partanen T, Valanne E. The infant cry: a spectrographic and auditory analysis. Clin Dev Med. 1968; 29(1):1-42.

18 - Lawrence J, Alcock D, McGrath P, Kay J, MacMurray SB, Dulberg C. The development of a tool to assess neonatal pain. Neonatal Netw. 1993; 12(6):59-66.

19 - Rosner B. Fundamentals of biostatistics. 2. ed. Boston: Duxbury Press; 1986.

20 - Branco A, Fekete SMW, Rugolo LMSS, Rehder MI. The newborn pain cry: descriptive acoustic spectrographic analysis. Int J Pediatr Otorhinolaryngol. (No prelo). 
21- McGrath PJ. Behavioral measures of pain. In: Finley GA, McGrath PJ. Measurement of pain in infants and children, progress in pain research and management. v. 10. Seattle: IASP Press; 1998.

22 - Guinsburg R. Avaliação e tratamento da dor no recém-nascido. J Pediatr. 1999; 79(3):149-60.

23 - Golub HL, Corwin MJ. Infant cry: a clue to diagnosis. Pediatrics. 1982; 69(2):197-201.

24 - Fort A, Manfredi C. Acoustic analysis of newborn infant cry signals. Med Eng Phys. 1998; 20(6):432-42. 25 - Fuller BF. Acoustic discrimination of three types of infant cries. Nurs Res. 1991; 40(3):156-60.

26 - McGrath PA. An assessment of children's pain: a review of behavioral, physiological and direct scaling techniques. Pain. 1987; 31(2):147-76.

27 - Beyer JE, Wells $N$. The assessment of pain in children. Pediatr Clin North Am. 1989; 36(4):837-54. 28 - Fort A, Ismaelli A, Manfredi C, Bruscaglioni P. Parametric and non-parametric estimation of speech formants: application to infant cry. Med Eng Phys. 1996; 18(8):677-91.
29 - Michelsson K, Järvenpää AL, Rinne A. Sound spectrographic analysis of pain cry in preterm infants. Early Hum Dev. 1983; 8(2):141-9.

30 - Bellieni CV, Sisto R, Cordelli DM, Buonocore G. Cry features reflect pain intensity in term newborns: an alarm threshold. Pediatr Res. 2004; 55(1):142-6. 31 - Porter FL, Porges SW, Marshall RE. Newborn pain cries and vagal tone: parallel changes in response to circumcision. Child Dev. 1988; 59(2):495-505. 32 - Michelsson K, Eklund K; Leppänen P; Lyytinen $\mathrm{H}$. Cry characteristics of 172 healthy 1-to 7-day-old infants. Folia Phoniatr Logop. 2002; 54(4):190-200.

33- Corwin MJ, Lester BM, Golub HL. The infant cry: what can it tell us? Curr Probl Pediatr. 1996; 26(9):325-34.

34 - Fuller BF, Horii Y. Differences in fundamental frequency, jitter, and shimmer among four types of infant vocalizations. J Commun Disord. 1986; 19(6):441-7.

35 - Porter FL, Miller RH, Marshall RE. Neonatal pain cries: effect of circumcision on acoustic features and perceived urgency. Child Dev. 1986; 57(3):790-802.

RECEBIDO EM: 07/08/06

ACEITO EM: 31/10/06

Endereço para Correspondência:

Rua João Marques, 82

Tietê - SP

CEP: $18530-000$

Tel/Fax: (15) 32826725

E-mail: anete.branco@uol.com.br 